\title{
Intermediate bronchial fistula caused by mediastinal drainage tube compression and fungal infection: a case report
}

\author{
Yahua Li ${ }^{1}$ Kewei Ren ${ }^{1}$, Liqun $\mathrm{Ye}^{2}$, Jianzhuang Ren ${ }^{1}$ and Xinwei Han ${ }^{1 *}$ (D)
}

\begin{abstract}
Background: Intermediate bronchial fistula formation caused by mediastinal drainage tube compression and fungal infection is rare.

Case presentation: A 50-year-old male patient with type 2 diabetes was observed air filling in mediastinal drainage tube, 12 days after esophagectomy for esophageal squamous carcinoma. Based on the results of computed tomography, bronchoscopy and pathology, the diagnosis of intermediate bronchial fistula caused by mediastinal drainage tube compression and fungal infection was made. Anti-fungal drug and temporary covered metallic stent was used. After stent removed, the fistula was healed with some granulation hyperplasia. He was free from respiratory symptom during 1 year follow-up.

Conclusion: Intermediate bronchial fistula caused by the combination of mediastinal drainage tube compression and fungal infection is rare. Timely stenting could boost the healing of fistula via granulation tissue proliferation.
\end{abstract}

Keywords: Tracheal stent, Intermediate bronchial fistula, Fungal infection

\section{Background}

Intermediate bronchial fistula formation caused by mediastinal drainage tube compression and fungal infection is rare. Stent placement as a minimally invasive therapeutic option may be recommend in such cases. Usually, silicon stent placement is the first choice. However, placement of small diameter silicon stent in small- to medium-caliber airway is not available in some regions. Here, we introduce the experience of small diameter individualized Y-shaped covered self-expandable metallic stent (SEMS) for intermediate bronchial fistula.

\section{Case presentation}

A 50-year-old male patient with type 2 diabetes was observed air filling in mediastinal drainage tube, 12 days after esophagectomy for esophageal squamous carcinoma. Chest computed tomography presented normal anastomosis. From the lung window of chest multi-slice $\mathrm{CT}$, the connection of intermediate bronchus and

\footnotetext{
* Correspondence: fcchanxw@zzu.edu.cn

${ }^{1}$ Department of Interventional Radiology, The First Affiliated Hospital of Zhengzhou University, Zhengzhou 450052, People's Republic of China Full list of author information is available at the end of the article
}

mediastinal drainage tube tract was observed (Fig. 1). Bronchoscopy revealed massive white necrotic materials attached the wall of intermediate bronchus. Distal intermediate bronchus was compressed (Fig. 2). After biopsy was harvested, the end of mediastinal drainage tube emerged and located in the center of necrotic materials (Fig. 3). Biopsy result is depicting numerous fungal hyphae (Fig. 4). Benign Intermediate bronchial fistula and fungal infection were diagnosed.

Silicon stent was the first choice for benign tracheobronchial fistula. However, small diameter Y-shaped silicon stent placement in second carina is not available in our hospital. An individualized Y-shaped covered selfexpandable metallic stent (SEMS) temporary placement and antifungal treatment were developed. Initially, stent was designed depending on the diameter and length measurement result of right main bronchus, right upper bronchus and intermediate bronchus from CT. The diameter of bronchial part, upper lobe part and intermediate bronchial part is $14 \mathrm{~mm}, 10 \mathrm{~mm}$ and $12 \mathrm{~mm}$. The length is $10 \mathrm{~mm}, 10 \mathrm{~mm}$ and $25 \mathrm{~mm}$, respectively. Under the guidance of fluoroscopy, two super-stiff guide wires were exchanged into the right lower and right 


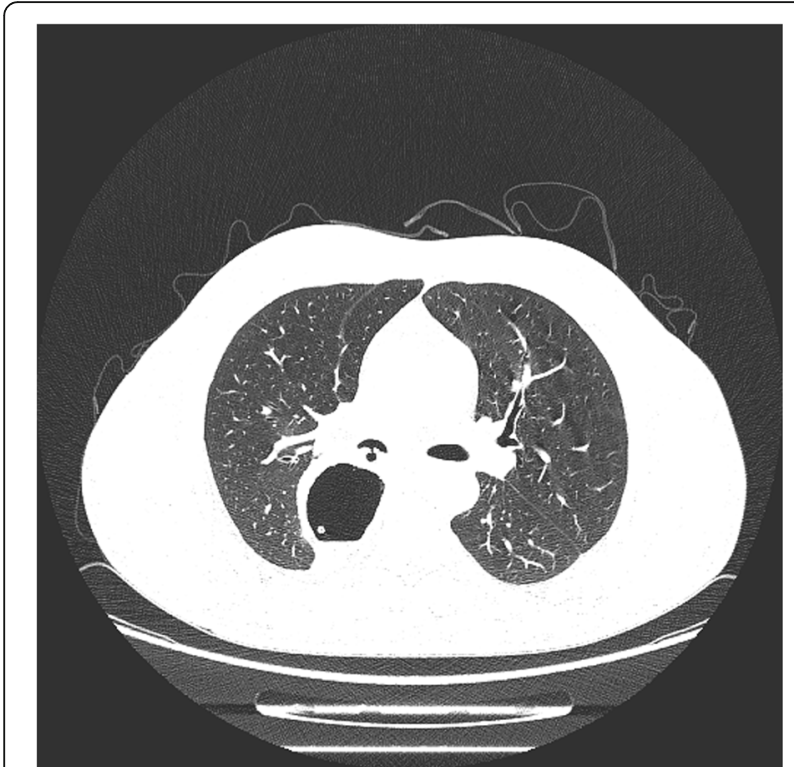

Fig. 1 A connection between intermediate bronchus and mediastinal drainage tube tract was observed through lung window

upper lobe bronchi. Once the delivery system that contained Y-shaped covered SEMS in place, the stent was released (Fig. 5). No procedure related complications happened. Bronchoscopy were performed to demonstrate the closure of fistula (Fig. 6). Mediastinal drainage tube was withdrawn gradually. Forty days later, CT shows the fistula tract was cured (Fig. 7). The stent was successfully removed. Bronchoscopy revealed the fistula

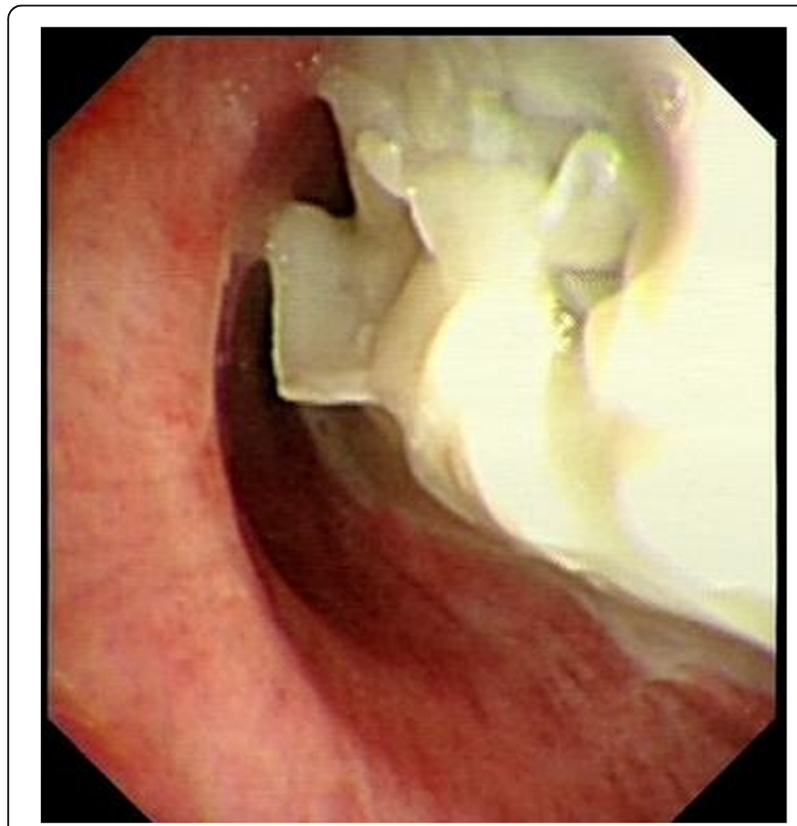

Fig. 2 Bronchoscopy revealed that massive white necrotic materials attached the wall of intermediate bronchus

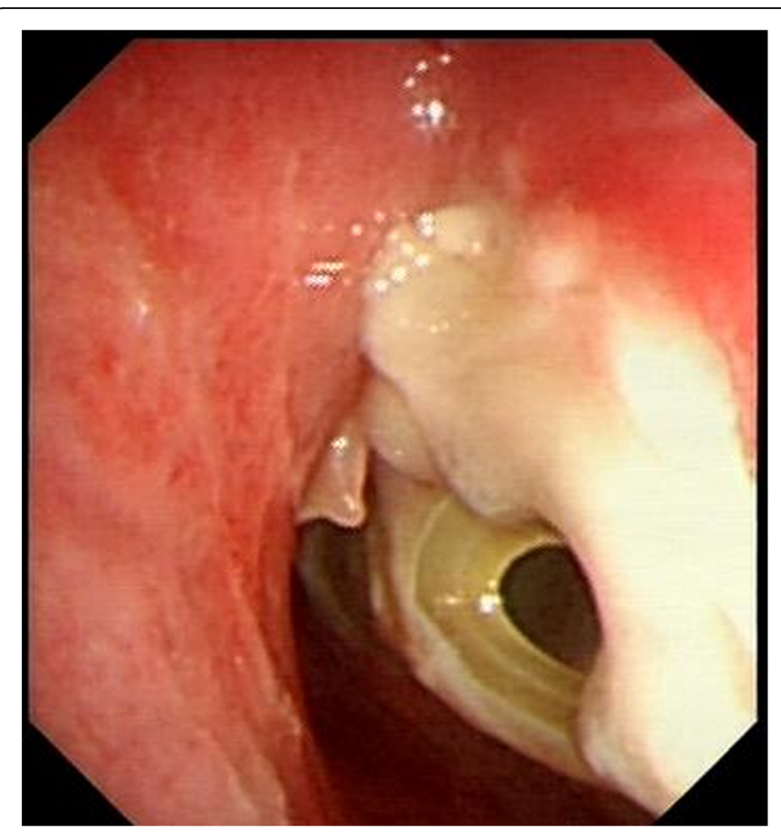

Fig. 3 After biopsy was harvested, the end of mediastinal drainage tube emerged and located in the center of necrotic materials. Distal intermediate bronchus was compressed

was healed with some granulation hyperplasia (Fig. 8). He was free from respiratory symptom during 1 year follow-up.

\section{Discussion and conclusions}

Intermediate bronchial fistula formation caused by mediastinal drainage tube compression and fungal infection is rare. The major risk factor for fungal infection is immunodeficiency including transplantation, AIDS, prolonged therapy with corticosteroids and malignancy et.al. Uncontrolled diabetes mellitus could results mildly immunosuppression and increases the risk of bacterial infection. In our case, the combination of mediastinal drainage tube compression and destroy effect of fungal infection contribute to bronchial fistula formation.

Imaging findings are crucial for the diagnosis. From the lung window of chest multi-slice $\mathrm{CT}$, the connection of intermediate bronchus and mediastinal drainage tube tract was observed. The specific finding of fungal infection is pseudomembrane. Fungal infection would bring completely demolished mucosa, transmural bronchial necrosis and pseudomembranes. The biopsy results depicting numerous fungal hyphae. Based on the evidence above, the diagnosis of benign intermediate bronchial fistula was made.

Early diagnosis and antifungal treatment may significantly improve the outcome. Arguder and colleagues reported a case of pseudomembranous aspergillus tracheobronchities, which caused tracheal perforation [1]. The patient was unsuitable for any invasive 

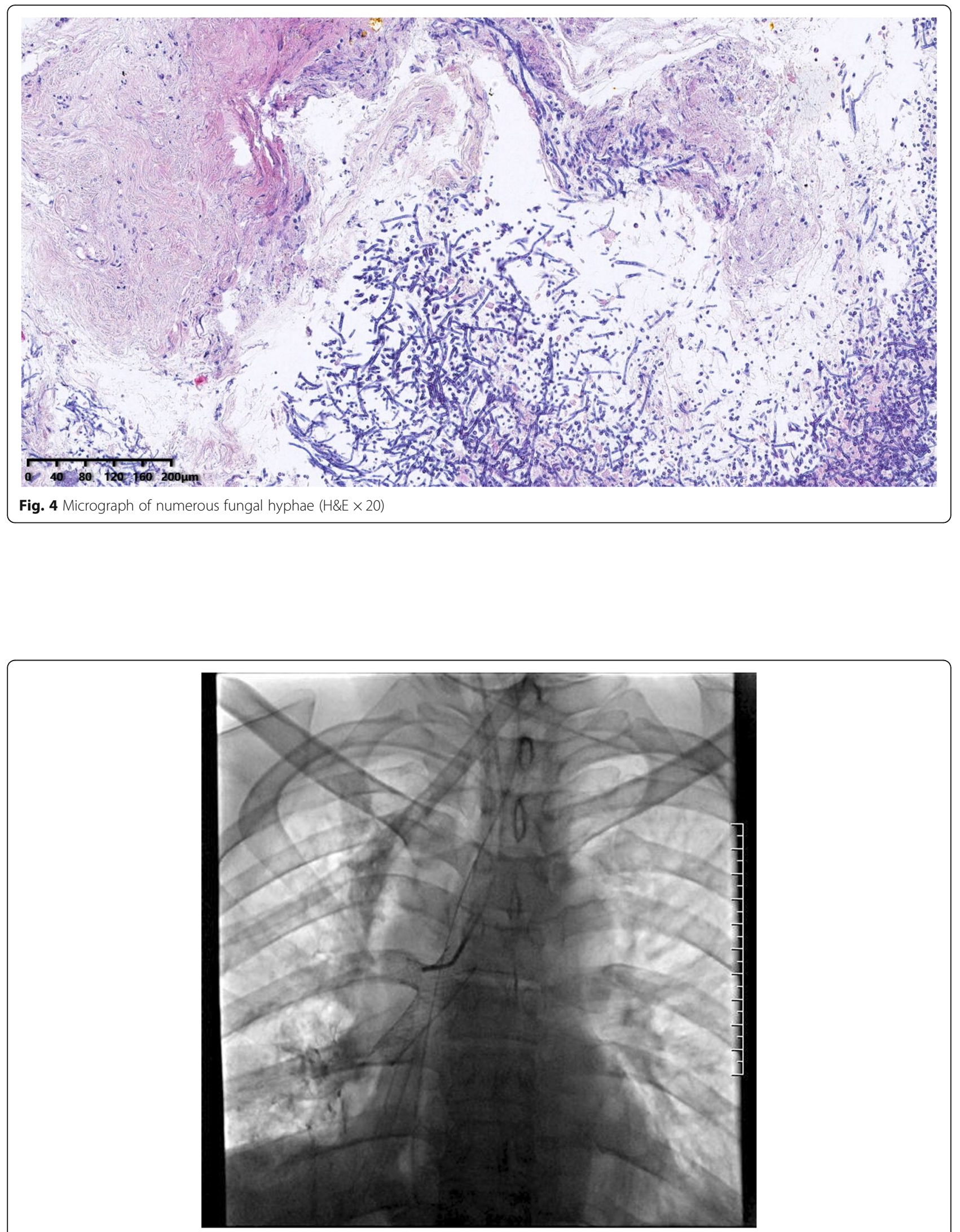

Fig. 5 The stent was placed under the guidance of fluoroscopy 


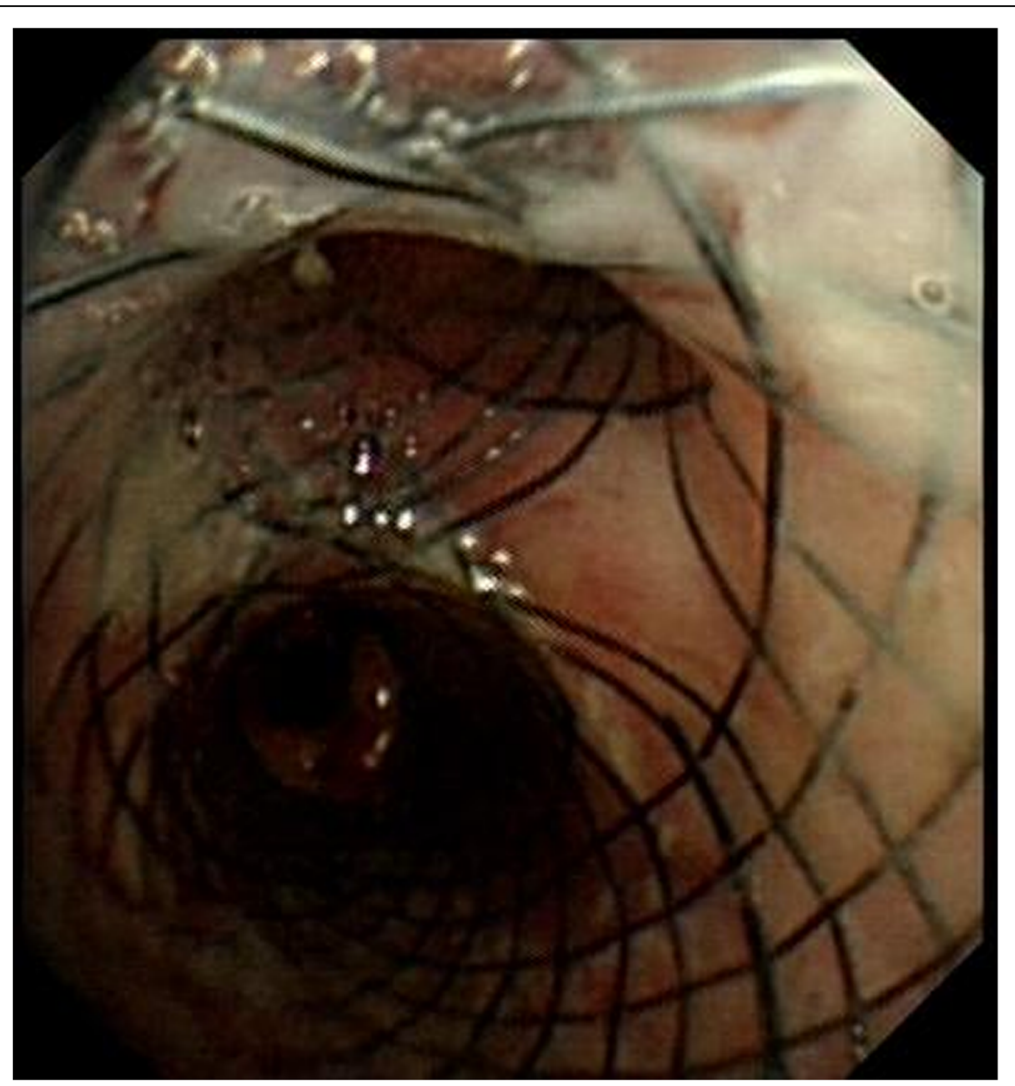

Fig. 6 Bronchoscopy demonstrate the closure of fistula

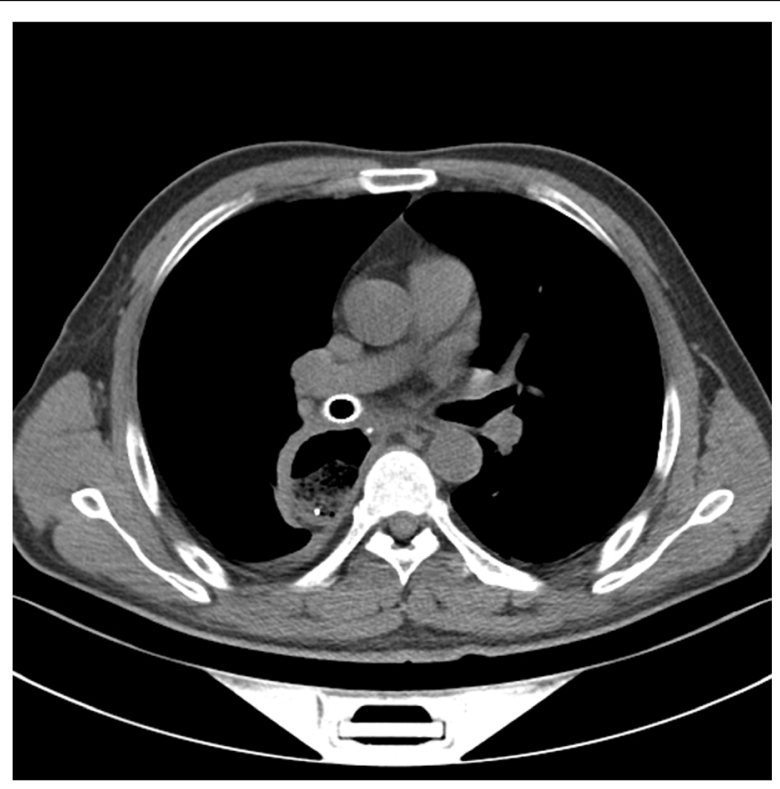

Fig. $7 \mathrm{CT}$ shows the fistula tract was cured

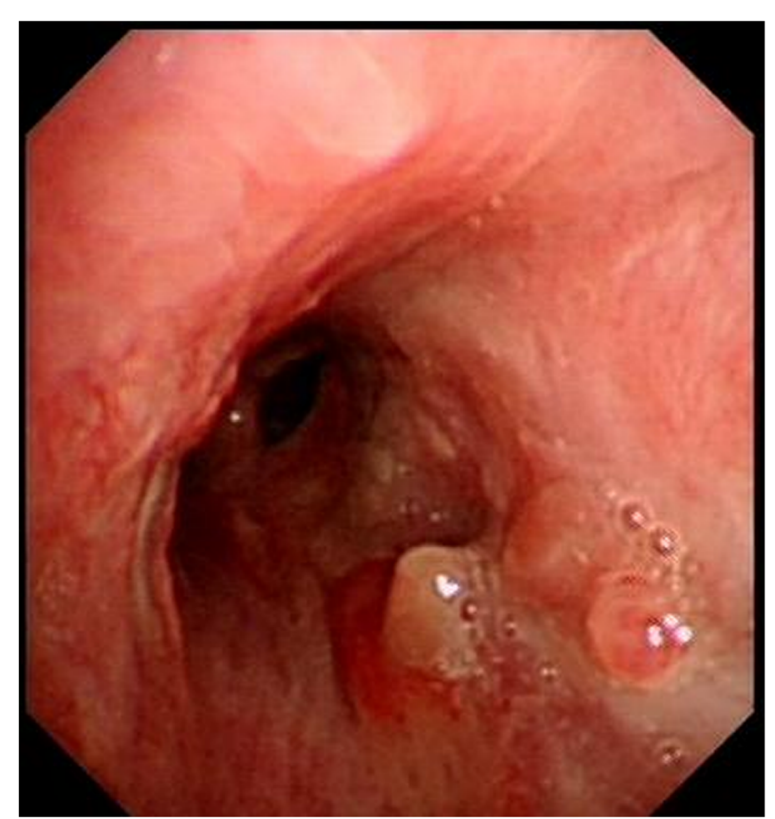

Fig. 8 After stent remove, fistula was healed with some granulation hyperplasia 
procedure because of very large perforation and died of respiratory failure. In our case, due to the fistula in intermediate bronchus, the lung function is not affect obviously. After the diagnosis of fungal infection from pathology was made, antifungal drug was given.

The treatment strategies of benign tracheobronchial fistula including surgery and endobronchial stenting. Considering the general condition of our patient, endobronchial stenting as a minimally invasive was developed. Small diameter silicon stent placement was the first choice in our case, but it is not available in our hospital, only metallic stent can be used. Although FDA warning uncovered metallic stenting in benign tracheal stenosis due to granulation hyperplasia. The application of covered metallic stent benefit many patients with tracheal fistula and stenosis [2-4]. The obstacles of the early years of metallic stent implantation involved the limitations of stent sizes. Ill-matched stent including undersized stent and oversized stent may cause undue friction and pressure, result in excessive granulation tissue formation [5]. To minimize the complications of granulation tissue formation after metallic stent placement, we ordered an individualized covered metallic stent to match the diameter of bronchus. Poststent 40 days, sputum retention was observed through bronchoscopy, no stent migration and severe granulation hyperplasia happened.

Thus, we conclude that: 1 . Tracheobronchial fistula caused by the combination of mediastinal drainage tube compression and fungal infection is rare. It is characterized by pseudomembranous formation on the wall of airway. 2. Uncontrolled diabetes mellitus could results mildly immunosuppression and increases the risk of bacterial infection. 3. Timely stenting could boost the healing of fistula via granulation tissue proliferation.

\section{Abbreviations}

CT: Computed tompgraphy; SEMS: Self-expandable metallic stent

\section{Acknowledgements}

Not applicable.

\section{Authors' contributions}

(I) Administrative support: YL \& KR; (II) Operation: All authors; (IV) Data collection and follow-up: All authors; (V) Manuscript writing: YL; (VI) Final approval of manuscript: All authors. All authors read and approved the final manuscript.

\section{Funding}

Not applicable.

Availability of data and materials

Please contact author for data requests.

Ethics approval and consent to participate

Not applicable.

Consent for publication

Not applicable.
Competing interests

The authors declare that they have no competing interests.

\section{Author details}

${ }^{1}$ Department of Interventional Radiology, The First Affiliated Hospital of Zhengzhou University, Zhengzhou 450052, People's Republic of China.

${ }^{2}$ Department of Respiratory Medicine, The First Affiliated Hospital of

Zhengzhou University, Zhengzhou 450052, People's Republic of China.

Received: 15 August 2019 Accepted: 28 October 2019

Published online: 07 November 2019

References

1. Arguder E, Senturk A, Hasanoglu HC, et al. Unique case of pseudomembranous aspergillus tracheobronchitis: tracheal perforation and Horner's syndrome. Mycopathologia. 2016;181(11-12):885-9.

2. Cao M, Zhu Q, Wang W, et al. Clinical application of fully covered selfexpandable metal stents in the treatment of bronchial fistula. Thorac Cardiovasc Surg. 2016;64(6):533-9.

3. Li TF, Duan XH, Han XW, et al. Application of combined-type Y-shaped covered metallic stents for the treatment of gastrotracheal fistulas and gastrobronchial fistulas. J Thorac Cardiovasc Surg. 2016;152(2):557-63.

4. Ma J, Han X, Wu G, et al. Outcomes of temporary partially covered stent placement for benign tracheobronchial stenosis. Cardiovasc Intervent Radiol. 2016;39(8):1144-51.

5. Avasarala SK, Freitag L, Mehta AC. Metallic endobronchial stents: a contemporary resurrection. Chest. 2019;155(6):1246-59.

\section{Publisher's Note}

Springer Nature remains neutral with regard to jurisdictional claims in published maps and institutional affiliations. 